\title{
Surgery in elderly cancer patients
}

\author{
Peter T Scardino
}

Over the last century, life expectancy has been steadily rising. As recently as 2003, the US Vital Statistics estimated median life expectancy for 65-year-old men as 17 years; for 70-year-olds it was 13 years, for 75 -year-olds it was 9 years, and for 80-year-olds it was 7 years. In developed countries, men and women over 75 years of age comprise the most rapidly growing segment of the population. By 2020 , there are expected to be over 6 million octogenarians in the US, and by the middle of this century, a million centenarians. Major causes of disability are declining steadily, leaving many more elderly people living active, self-sufficient lives.

Cancer is a disease of aging, with some $80 \%$ of cases diagnosed in people aged over 55 years. While the mortality rate from cancer remains stable, the incidence of cancer is rising. As the elderly population increases, we will see a dramatic rise in the number of new cancer cases each year. Urologists will be faced with elderly patients who have increasing numbers of curable cancers. There are abundant data to suggest that elderly patients (over 65 years of age) are less likely to receive optimal therapy for cancer, even when they are in otherwise good health. Weizer et al. discussed the outcomes of radical cystectomy and urinary diversion for patients with bladder cancer, confirming once again that the mortality rate for this procedure is no greater in properly selected elderly patients than in younger patients (Weizer et al. [2006] Nat Clin Pract Urol 3: 346-347). Several large studies have also reported radical cystectomy with very low mortality rates in elderly patients, even in those with substantial comorbidities. Cystectomy, along with adjuvant chemotherapy, has also been shown to significantly prolong the survival of patients with bladder cancer, even among the elderly, compared with more conservative measures.
By relying on

outmoded

assumptions... urologists

might be

denying older

patients...

therapy

that could

substantially

prolong their

lives.

PT Scardino is the Editor-in-Chief of Nature Clinical Practice Urology.

Competing interests

The author declared he has no competing interests.

www.nature.com/clinicalpractice doi:10.1038/ncpuro0557
Performing radical surgery for cancer in elderly patients is associated with an increased risk of perioperative complications. Perioperative mortality after radical prostatectomy in patients over 65 years of age, for example, is about $0.5 \%$ higher than in younger men. Yet morbidity and mortality are not associated so much with age as with the severity of comorbidities (Dillioglugil O et al. [1997] J Urol 157: 1760-1767). Elderly patients generally tolerate radical surgery well, even though they might tolerate complications poorly. It is important to evaluate candidates for surgery thoroughly before the operation and to take measures to optimize organ function and minimize the risk of complications. In order to do this, the entire surgical team-nursing staff, anesthesiologists, consultants and surgeonsneed to be familiar with the individual patient's pre-existing comorbidities and risk factors.

While some surgeons set age limits of 65 years or over as a contraindication to radical prostatectomy, a randomized trial from Sweden reported a significant overall and cancer-specific survival advantage compared to watchful waiting for men with clinically localized prostate cancer within 6-7 years of surgery (Bill-Axelson A et al. [2005] N Engl J Med 352: 1977-1984). With longevity increasing and disabilities associated with advancing age decreasing, the benefits of cancer control with radical prostatectomy in older men should be weighed against the increased risks of perioperative complications and the effects on urinary, bowel and sexual function.

By relying on outmoded assumptions about the value of active cancer treatment in the elderly, urologists might be denying older patients the opportunity for radical therapy that could substantially prolong their lives. The time has come to rethink our management of urologic cancers in the elderly population. 